\title{
The Role of Dynamic Magnetic Resonance Imaging in the Evaluation of Pulmonary Nodules and Masses
}

\author{
Fatih Alper $^{\mathrm{a}}$ Adile Tuba Polat Kurt ${ }^{\mathrm{a}}$ Yener Aydin ${ }^{\mathrm{b}} \quad$ Mesut Ozgokce $^{\mathrm{a}}$ \\ Metin Akgunc \\ Departments of a Radiology, ${ }^{\mathrm{b}}$ Thoracic Surgery and ${ }^{\mathrm{c} C h e s t ~ D i s e a s e s, ~ F a c u l t y ~ o f ~ M e d i c i n e, ~ A t a t u r k ~ U n i v e r s i t y, ~}$ \\ Erzurum, Turkey
}

\section{Key Words}

Pulmonary nodules $\cdot$ Dynamic MRI $\cdot$ Kinetic parameters

\begin{abstract}
Objective: The aim of our study was to determine whether or not dynamic magnetic resonance imaging (MRI) with kinetic and morphological parameters can reveal significant differences between malignant and benign pulmonary lesions, and thus to evaluate the use of dynamic MRI in the management of pulmonary nodules. Patients and Methods: Thirty-one patients (4 women and $27 \mathrm{men}$ ) underwent 1.5 T MRI, where 10 consecutive dynamic series were performed every $30 \mathrm{~s}$ by using 3D fast low-angle shot sequences. The percentage increase in the signal intensity of the lesions was determined for each time point. Time-enhancement curves of the lesions were drawn and classified into four types: A, B, C and D. Early peak (EP) and maximum peak (MP) values of the curves were calculated and compared with the diagnoses of the patients. The usefulness of these parameters was tested statistically. In addition to the comparison of the parameters between the groups, receiver-operating characteristic analysis was used to assess sensitivity, specificity, and both positive and negative predictive values of EP and MP parameters. Results: Of the 31 pulmonary le-
\end{abstract}

sions, 16 (52\%) were malignant. These showed a stronger enhancement with higher median values of EP and MP (77.08 and 123.15 , respectively) than those corresponding to the benign lesions (14.45 and 32.53, respectively). There were significant differences between the benign and malignant lesions $(p<0.001)$. Sensitivity, specificity, positive predictive value and negative predictive value were $75,93,92$ and $78 \%$ for EP and 93, 86, 88 and 93\% for MP, respectively. Conclusion: A combination of kinetic and morphological evaluation in dynamic MRI provided accurate differentiation between benign and malignant pulmonary lesions. It was a useful and noninvasive method of evaluating pulmonary nodules.

Copyright $\odot 2012$ S. Karger AG, Basel

\section{Introduction}

Indeterminate lesions are still one of the most challenging problems in current clinical practice. In such cases, computerized tomography (CT) serves as the standard of reference and its use is recommended for the evaluation of these nodules in the current guidelines [1-8]. Although magnetic resonance imaging (MRI) is not used as a first choice in the evaluation of indeterminate nodules,

\section{KARGER}

Fax +41 613061234

E-Mail karger@karger.ch

www.karger.com
(C) 2012 S. Karger AG, Basel

$1011-7571 / 13 / 0221-0080 \$ 38.00 / 0$

Accessible online at:

www.karger.com/mpp
Dr. Yener Aydin

Department of Thoracic Surgery, Faculty of Medicine, Ataturk University

TR-252400 Erzurum (Turkey)

Tel. +90 $4423166333 / 2181$

E-Mail dryeneraydin@ @otmail.com 
the advantages of using dynamic MRI for tumor catheterization have been demonstrated in several studies $[9$, 10], and due to technical improvements MRI has gained importance for tumor detection and interpretation [1113].

In this study, we aimed to determine whether MRI with kinetic and morphological parameters revealed significant differences between malignant and benign pulmonary lesions, and thus to evaluate the use of dynamic MRI in the management of indeterminate pulmonary lesions.

\section{Subjects and Methods}

\section{Case Selection}

This prospective study included 31 patients, 27 men and 4 women, from January 2009 to January 2010. They all signed informed consent forms. All the patients were diagnosed by direct chest X-ray and underwent a CT scan. The morphological characteristics of the lesions were evaluated. Dynamic MRI was performed on selected patients who required further evaluation. The 2 patients who could not tolerate MRI were excluded from the study.

\section{Imaging Technique}

MRI was performed with a 1.5 -tesla scanner using body coil (Magnetom Symphony; Siemens, Germany). The localizations of the lesions were detected by localizer images which were achieved in three planes. The images obtained in the sagittal and transverse planes at the greatest diameter were evaluated by dynamic MRI. Breath-hold fat-suppressed $\mathrm{T}_{1}$-weighted FLASH 3D (TR/TE: 6/2.51; FA: 10, voxel size: $2.3 \times 1.4 \times 5 \mathrm{~mm}$ ) was sequenced. Following the first images without contrast delivery, the contrast medium (i.v. $0.1 \mathrm{mmol} / \mathrm{kg}$ gadolinium-DPTA) was administered through an automatic injector with an 18- to 20-gauge cannula positioned in an antecubital vein at a flow rate of $3 \mathrm{ml} / \mathrm{s}$; about $15 \mathrm{ml}$ saline was injected following the gadolinium. Dynamic images were obtained at $30,60,90,120,150,180,210,240$ and $600 \mathrm{~s}$ with an 8 - to 12-second breath-holding episode per series.

\section{Evaluation of the Images: Morphological Evaluation}

The lesion size, contour, presence of cavity formation, intensity of adipose tissue and signal characteristics were also assessed using a $\mathrm{T}_{1}$-weighted gradient echo and a $\mathrm{T}_{2}$-weighted turbo spin echo. The signal characteristics (isointense, hypointense, hyperintense and heterogeneous) were established comparing thoracic wall images to muscle images. CT scans and direct X-ray images were compared to evaluate the size of the nodules.

\section{Kinetic Evaluation}

Signal intensity (SI) measurement was performed to evaluate the dynamic images by positioning the circular region of interest (ROI) at the most contrast-enhancing part of the lesion for each case. While establishing the size and region for the ROI, positioning it in the larger area was considered in order to minimize the effect of the region on hemodynamic inhomogeneity of the tumor without necrosis or calcification. In addition, any pulmonary parenchyma and pulmonary veins adjacent to the lesion area were excluded in order to avoid partial volume effect. The same physician positioned the ROI manually in the same region of the lesion in all images, and the SI measurement was performed from the peripheral solid area for the necrotic lesions. The area of the ROI varied between 0.23 and $1.60 \mathrm{~cm}^{2}$. After the SI measurement, the following equation was used to establish the ratio increase (SI\%) for each time point $(\mathrm{t}): \mathrm{SI} \% \mathrm{t}=[(\mathrm{SIt}-\mathrm{SIt} 0) / \mathrm{SIt} 0] \times 100$. $\mathrm{t} 0$ refers to the value before the injection of the contrast medium.

Time-SI curves were converted to time-contrast curves (SI\%) for each lesion using this equation. These curves were evaluated using the classification of Schaefer et al. [14]. This classification includes type A: a strong increase in SI within the first minute followed by a rapid decrease through the early peak (EP); type B: a strong increase in SI within the first minute followed by a plateau or slow increase without washout; type C: a moderate or mild increase in SI without a rapid EP value, and type D: no significant increase in SI (those with a mean increase of $<10 \%$ were included in this group).

The other parameters evaluated were the EP value, i.e. the maximum relative contrast level within the 1st minute following $\mathrm{t} 0$, and the maximum peak (MP) value, i.e. the maximum relative contrast level during the procedure.

Statistical Analysis

Statistical analysis was performed using frequency distribution. The $\chi^{2}$ test, Fisher's exact test and the Mann-Whitney U test were used to compare variables between the groups. Also, receiver-operating characteristic (ROC) analysis was used to assess sensitivity, specificity, and both positive and negative predictive values of the EP and MP parameters. SPSS 17.0 software was used to analyze the data.

\section{Results}

Of the 31 patients, 15 (48\%) were diagnosed with benign and $16(52 \%)$ with malignant lesions. The overall mean age was $57 \pm 12$ years (range: $40-70$ years). The mean age of the patients with benign lesions was $55 \pm 14$ years, while that of patients with malignant lesions was $60 \pm 9$ years and the difference was not statistically different $(\mathrm{p}>0.05)$.

The mean size of the benign lesions was $26.5 \pm 5.2$ $\mathrm{mm}$ (range: 11-59 $\mathrm{mm}$ ), while the mean size of the malignant lesions was $48 \pm 21.2 \mathrm{~mm}$ (range: $21-92 \mathrm{~mm}$ ); the longest diameter was measured on the axial images. The difference in size between the benign and malignant lesions was statistically significant $(\mathrm{p}<0.05)$. Five $(33 \%)$ of the benign lesions were $\geq 30 \mathrm{~mm}$, while $3(19 \%)$ of the malignant lesions were $<30 \mathrm{~mm}$.

A total of 8 benign lesions had a smooth surface, while 6 had a lobulated contour. Three of the malignant lesions were lobulated, 5 were irregular, and 8 had a spiculated 


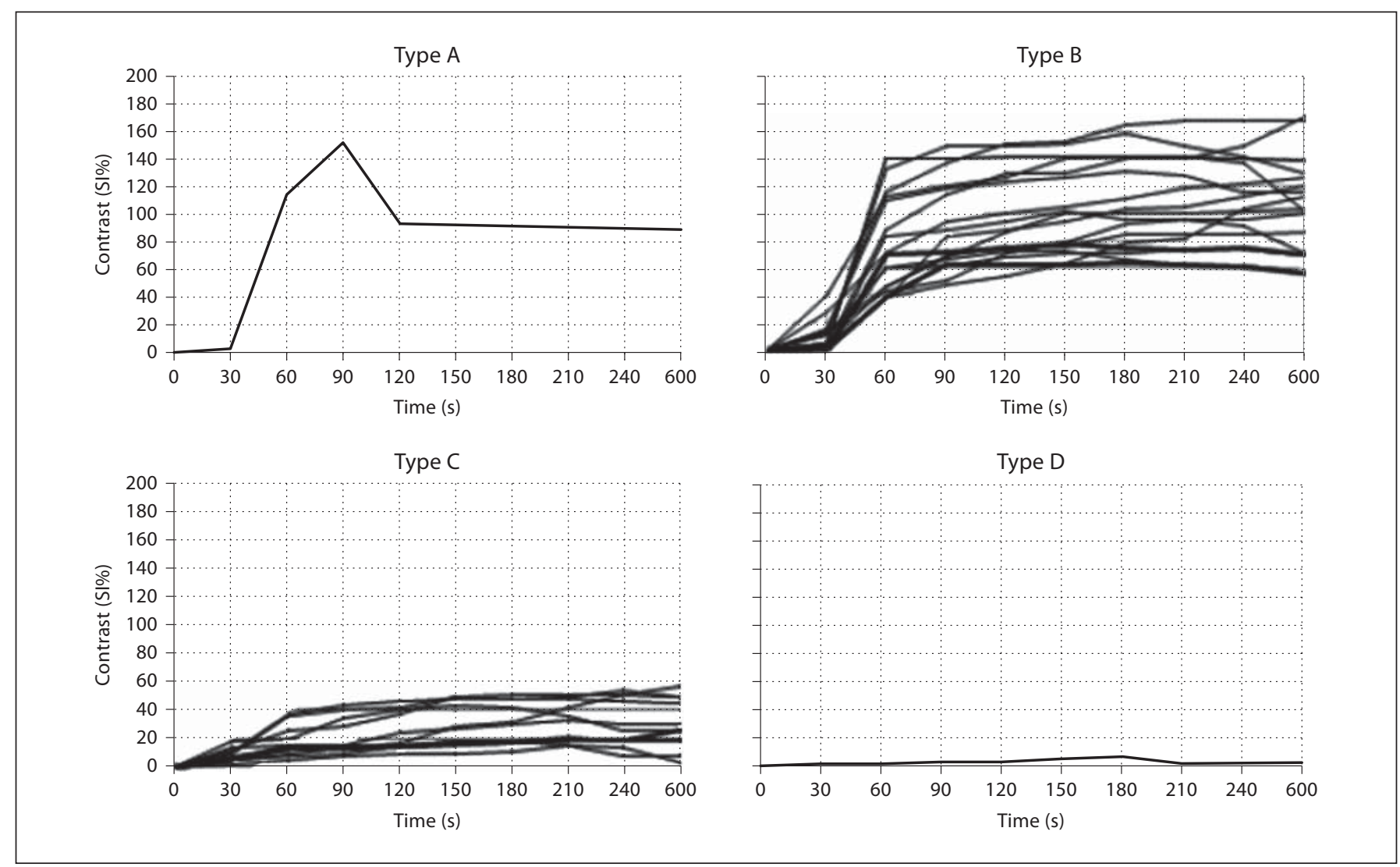

Fig. 1. The distribution of the 4 different patterns (types A, B, C and D) according to time-contrast enhancement curves.

border. In addition, all lesions diagnosed with squamous cell carcinoma were irregular with spiculated borders. On the other hand, 2 lesions with a lobulated contour were considered adenocarcinoma, while 1 was considered large cell carcinoma.

Of the 16 patients with malignant lesions, 8 (50\%) underwent CT-guided transthoracic needle aspiration biopsy, and 5 (31\%) underwent transbronchial biopsy. In addition, 3 (19\%) patients were diagnosed by thoracoscopic wedge resection. The number of patients diagnosed with squamous cell carcinoma was 8 (50\%), which included 6 (38\%) adenocarcinoma, 1 (6\%) non-small cell carcinoma and $1(6 \%)$ large cell carcinoma. Of the 15 patients with benign lesions, 6 (40\%) were diagnosed histopathologically. Of these, 4 patients had granuloma including 1 with tuberculoma, while 2 had hamartoma. The remaining 9 (60\%) patients with benign lesions were diagnosed by means of clinicoradiological features as well as by the findings of direct X-ray, CT and MRI during a 2-year follow-up period.
In the $\mathrm{T}_{1}$-weighted images, heterogeneous (4 vs. 1 ) and isointense signals ( 11 vs. 9) were higher in the malignant lesions whereas hypointense ( 2 vs. 0 ) and hyperintense signals ( 3 vs. 1 ) were seen in the benign ones. In the $\mathrm{T}_{2^{-}}$ weighted images, the heterogeneous signal was higher in the malignant lesions ( 9 vs. 4 ), whereas the hyperintense signal was higher in the benign ones (11 vs. 6). There was no isointense signal in the benign lesions and only 1 in the malignant ones. In both images, there was no statistical difference between the malignant and benign lesions in terms of signal intensities $(\mathrm{p}=0.179$ and $\mathrm{p}=0.113$ for $\mathrm{T}_{1^{-}}$and $\mathrm{T}_{2}$-weighted images, respectively). Lack of benefit of signal characteristics on differential diagnosis resulted in the necessity of further dynamic evaluation. Timecontrast enhancement curves obtained from the 10 consecutive dynamic series of pulmonary lesions and interpreted using the equation SI\%t $=[($ SIt - SIt 0$) /$ SIt 0$] \times 100$ are shown in figure 1 based on the disease type.

Only 1 patient with malignant disease showed the type A pattern. Fourteen patients with malignant dis- 

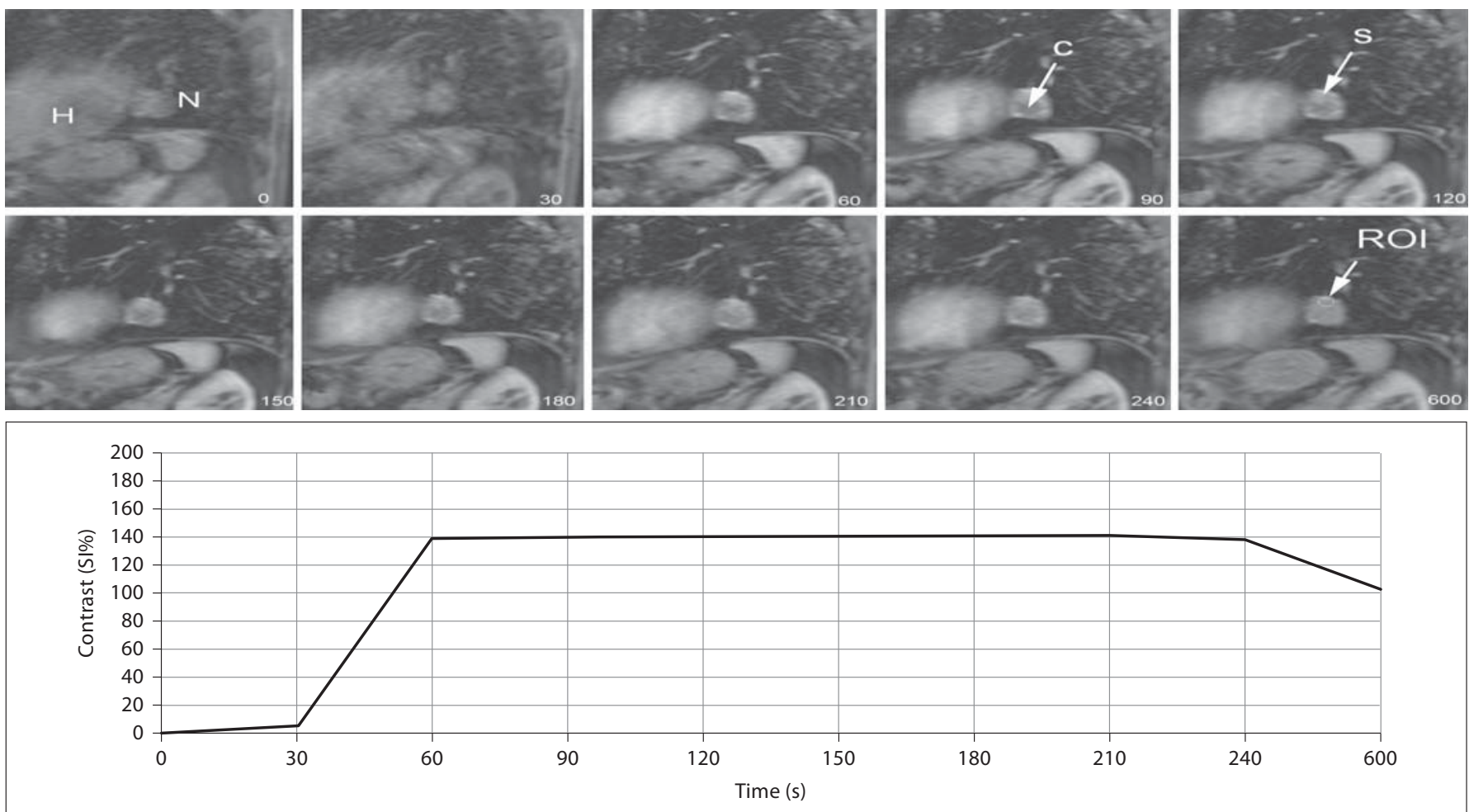

Fig. 2. Dynamic images of a 63 -year-old man showing a $29 \times 25 \mathrm{~mm}$ nodule $(\mathrm{N})$ with lobulated contour adjacent to the heart $(\mathrm{H})$, with a significantly increased SI ratio in the early phase. The nodule also has cystic (C) and necrotic components. Time-contrast enhancement curve showing type B pattern characterized by a strong increase in SI within the first minute with EP. The patient was diagnosed with adenocarcinoma. S = Solid.

Table 1. The proportion of malignant and benign lesions based on the dynamic curve types

\begin{tabular}{lllll}
\hline Diagnosis & Type A $(\mathrm{n}=1)$ & Type B $(\mathrm{n}=17)$ & Type C $(\mathrm{n}=11)$ & Type D $(\mathrm{n}=2)$ \\
\hline Malignant & $1(100 \%)$ & $14(82 \%)$ & $1(9 \%)$ & $0(0 \%)$ \\
Squamous cell carcinoma & 1 & 6 & 1 & 0 \\
Adenocarcinoma & 0 & 6 & 0 & 0 \\
Small cell carcinoma & 0 & 1 & 0 & 0 \\
Large cell carcinoma & 0 & 1 & 0 & 0 \\
Benign & $0(0 \%)$ & $3(18 \%)$ & $10(91 \%)$ & $2(100 \%)$ \\
Hamartoma & 0 & 1 & 1 & 0 \\
Granuloma & 0 & 2 & 1 & 1 \\
Follow-up diagnosis of benign cases & 0 & 0 & 8 & 1 \\
\hline
\end{tabular}

ease and 3 with benign disease showed the type B pattern. While 10 patients with benign disease and 1 with malignant disease showed the type C pattern, only 2 patients with benign disease showed the type D pattern. All diagnoses made and data based on the types of the curve are shown in table 1 . A higher proportion of the patients with malignant disease had type A and B curve patterns $(\mathrm{p}=0.001)$, while a higher proportion of the patients with benign disease had type $\mathrm{C}$ and $\mathrm{D}$ curve patterns with some overlapping of $\mathrm{B}$ and $\mathrm{C}$ curve patterns among the patients with benign and malignant lesions (fig. 2, 3). 

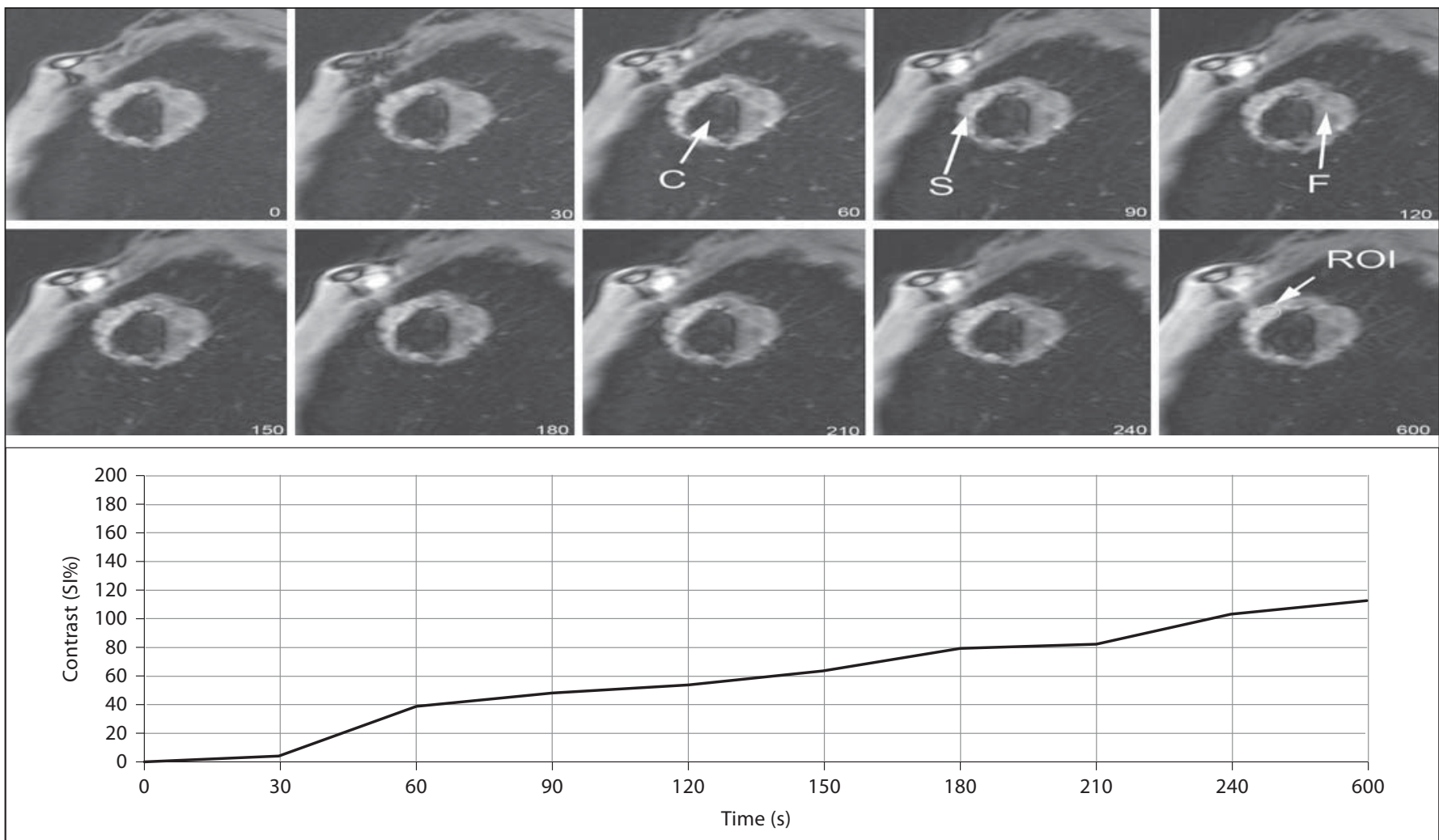

Fig. 3. Dynamic images of a 61-year-old man. Time-contrast enhancement curve showing EP within the first minute followed by a relatively slower increase in intensity through the peripheral cavitary lesion containing solid mass. The patient was diagnosed with squamous cell carcinoma. $\mathrm{C}=$ Cavitary; $\mathrm{S}=$ solid; $\mathrm{F}=$ fluid.

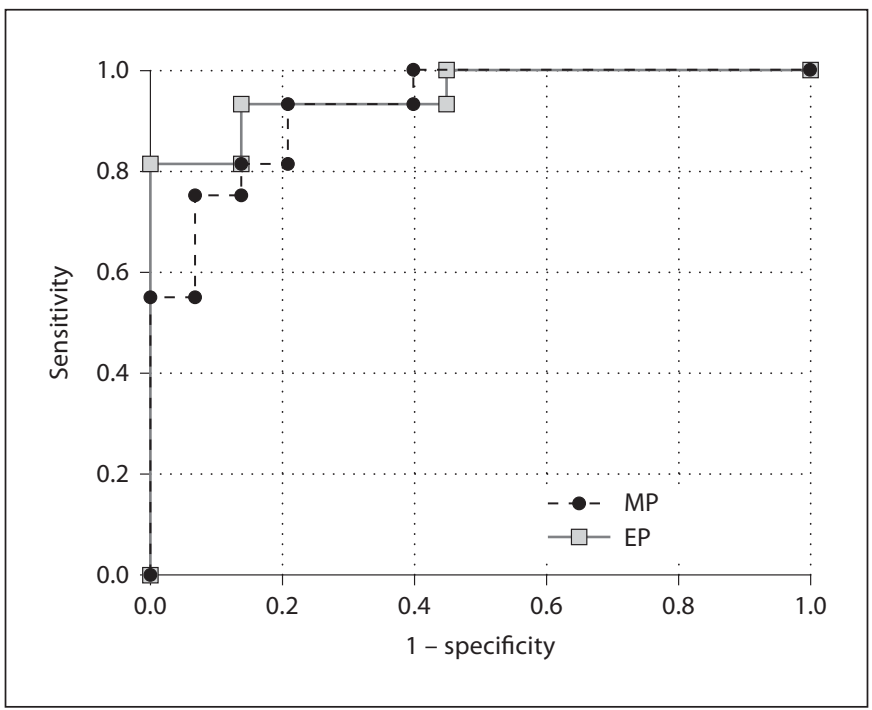

Fig. 4. ROC curves of MP and EP.
A comparison between the SI\% ratio of the steepest slope, EP, of the time-contrast enhancement curve within the first minute following injection and the maximum SI\% ratio, MP, in all dynamic series showed significant differences between benign and malignant disease. As a result, the patients with malignant disease had a significantly higher value of EP and MP ( $<<0.001)$. The median values of kinetic parameters in time-contrast enhancement curves are shown in table 2.

The ROC analysis showed a threshold value of $46.73 \%$ for EP with $75 \%$ sensitivity and $93 \%$ specificity. The analysis also showed a threshold value of $64.86 \%$ for MP with $93 \%$ sensitivity and $86 \%$ specificity. The area under the ROC curve was 0.929 and 0.954 for EP and MP, respectively, suggesting that the MP was more effective than the EP in differentiating between benign and malignant disease (fig. 4). 
Table 2. Time-contrast enhancement curve parameters

\begin{tabular}{lrrrrrl}
\hline $\begin{array}{l}\text { Param- } \\
\text { eter }\end{array}$ & \multicolumn{2}{l}{ Benign $(\mathrm{n}=15,48 \%)$} & & \multicolumn{2}{c}{ Malignant $(\mathrm{n}=16,52 \%)$} & $\mathrm{p}$ \\
\cline { 2 - 3 } & median & quartiles & & median & quartiles & \\
\hline EP & 14.45 & $7.75-37.50$ & & 77.08 & $44.68-113.70$ & $<0.001$ \\
MP & 32.53 & $17.24-56.60$ & & 123.15 & $101.00-149.90$ & $<0.001$ \\
\hline
\end{tabular}

\section{Discussion}

The findings of this study indicate that dynamic contrast-enhanced MRI in the management of indeterminate lesions was used to differentiate malignant from benign lesions and to evaluate lesion morphology, although there is no recommendation about its use in the algorithm of such lesions in current guidelines $[15,16]$.

It has been reported [17] that the maximum enhancement rate and slope as determined in the time-contrast enhancement curve correlated with tumor angiogenesis (microvascular density), while the washout correlated with tumor interstitium (the ratio of elastic and collagen fibers). Within this context, a rapid and strong uptake of contrast medium is associated with interstitial aggregation of the medium due to increased vascularity and capillary permeability. These findings are commonly associated with malignant tumors [18].

Washout of the contrast medium was found in $57 \%$ of patients with malignant nodules $(n=58)$ in a study by Schaefer et al. [14]. However, two other studies observed it only in inflammatory lesions and a carcinoid tumor, while there were no quantitative data regarding washout characteristics of pulmonary nodules $[19,20]$. In our study, we found that only 1 patient with malignant disease showed a type A curve characterized by a washout pattern. The biggest overlap was seen in the type B curve. Increased SI within the first minute was seen in both curve types even without washout, which was consistent with the data obtained from the literature, suggesting that an increased SI rate of the first passing of the contrast medium was associated with malignant lesions $[18,21$, 22]. However, most of the patients with benign disease showed the type C pattern characterized by a slow increase in SI without a rapid peak. Among the patients with malignant disease, only non-small cell carcinoma patients showed the type $\mathrm{C}$ pattern. This finding might be associated with low vascularity of the tumor.

In this study, kinetic analysis was performed using EP and MP values. As the concentration gradient between the tissue and blood after the first passing reaches the maximum level, a rapid increase is observed in the timecontrast enhancement curve parallel to tissue vascularization, resulting in acquiring quantitative data regarding tissue vascularization. In this study, EP values obtained at the steepest slope drawn within the first minute following bolus injection were compared. Significantly increased SI was observed in the malignant nodules compared to the benign ones. In addition, the MP values of the malignant lesions were higher $(p<0.001)$.

Although CT also allows quantification of tissue perfusion, it leads to exposure to an increased radiation dose without higher specificity [23-26]. Today, perfusion study with MRI is also possible without exposure to radiation $[27,28]$. Because the contrast media used in CT and MR do not have similar behavior, contrast enhancement values of both techniques are not directly comparable. The results of the studies using dynamic MRI have revealed a comparable sensitivity value with $\mathrm{CT}$, despite higher specificity $[14,20,21]$.

The limitations of our study were the small number of patients in the study group and probably the characteristics of the lesions. In this study only 1 lesion with type A was observed, but in our clinical practice we commonly encounter a type $B$ curve in patients with malignant lesions. Another limitation of our study was that we had no histopathological confirmation of some benign cases.

\section{Conclusion}

The results of our study suggest that MRI with kinetic and morphological analysis may reduce the ratio of resection of benign nodules as well as invasive procedures and confirm that MRI is applicable and repeatable in reducing the radiation burden. This technique is also used for multiple lesions, even those with different characteristics.

\footnotetext{
References

1 Araz O, Akgun M, Gorguner M: Lung cancer incidence in Erzurum city. EAJM 2010;42: 111-115.

2 Doganay S, Gocmen H, Yikilmaz A, Coskun A: Silicosis due to denim sandblasting in young people: MDCT findings. EAJM 2010; 42:21-23.

-3 Alper F, Akgun M, Onbas O, Araz O: CT findings in silicosis due to denim sandblasting. Eur Radiol 2008;18:2739-2744.
} 
4 Chang SY, Huang TC, Su WL, Chian CF, Perng WC: Multiple pulmonary nodules in ectopic adrenocorticotropic hormone syndrome: cause or result? Med Princ Pract 2012;21:292-294.

5 Al-Khawari H, Kovacs A, Athyal R, Al-Manfouhi H, Fayaz MS, Madda JP: Breast magnetic resonance imaging: initial experience in Kuwait. Med Princ Pract 2009;18:143148.

6 Brandman S, Ko JP: Pulmonary nodule detection, characterization, and management with multidetector computed tomography. J Thorac Imaging 2011;26:90-105.

7 Nair A, Hansell DM: European and North American lung cancer screening experience and implications for pulmonary nodule management. Eur Radiol 2011;21:24452454.

$\checkmark 8$ Beigelman-Aubry C, Hill C, Grenier PA: Management of an incidentally discovered pulmonary nodule. Eur Radiol 2007;17:449466.

9 Kono M, Adachi S, Kusumoto M, Sakai E: Clinical utility of Gd-DTPA-enhanced magnetic resonance imaging in lung cancer. J Thorac Imaging 1993;8:18-26.

10 Regier M, Kandel S, Kaul MG, Hoffmann B, Ittrich $\mathrm{H}$, Bansmann PM, Kemper J, NolteErnsting C, Heller M, Adam G, Biederer J: Detection of small pulmonary nodules in high-field MR at $3 \mathrm{~T}$ : evaluation of different pulse sequences using porcine lung explants. Eur Radiol 2007;17:1341-1351.

- 11 Vogt FM, Herborn CU, Hunold P, Lauenstein TC, Schröder T, Debatin JF, Barkhausen J: HASTE MRI versus chest radiography in the detection of pulmonary nodules: comparison with MDCT. AJR Am J Roentgenol 2004; 183:71-78.

$\checkmark 12$ Regier M, Schwarz D, Henes FO, Groth M, Kooijman H, Begemann PG, Adam G: Diffusion-weighted MR-imaging for the detection of pulmonary nodules at 1.5 Tesla: intraindividual comparison with multidetector computed tomography. J Med Imaging Radiat Oncol 2011;55:266-274.
13 Sieren JC, Ohno Y, Koyama H, Sugimura K, McLennan G: Recent technological and application developments in computed tomography and magnetic resonance imaging for improved pulmonary nodule detection and lung cancer staging. J Magn Reson Imaging 2010;32:1353-1369.

14 Schaefer JF, Schneider V, Vollmar J, Wehrmann M, Aebert H, Friedel G, Vonthein R, Schick F, Claussen CD: Solitary pulmonary nodules: dynamic contrast-enhanced MR imaging - perfusion differences in malignant and benign lesions. Radiology 2004; 232:544-553.

15 MacMahon H: Compliance with Fleischner Society guidelines for management of lung nodules: lessons and opportunities. Radiology 2010;255:14-15.

-16 Esmaili A, Munden RF, Mohammed TL: Small pulmonary nodule management: a survey of the members of the Society of Thoracic Radiology with comparison to the Fleischner Society guidelines. J Thorac Imaging 2011;26:27-31.

17 Fujimoto K, Abe T, Müller NL, Terasaki H, Kato S, Sadohara J, Kono R, Edamitsu O, Ishitake T, Hayashi A, Rikimaru T, Hayabuchi N: Small peripheral pulmonary carcinomas evaluated with dynamic MR imaging: correlation with tumor vascularity and prognosis. Radiology 2003;227:786-793.

18 Fontanini G, Vignati S, Boldrini L, Chinè S, Silvestri V, Lucchi M, Mussi A, Angeletti CA, Bevilacqua G: Vascular endothelial growth factor is associated with neovascularization and influences progression of non-small cell lung carcinoma. Clin Cancer Res 1997;3: 861-865.

19 Hittmair K, Eckersberger F, Klepetko W, Helbich T, Herold CJ: Evaluation of solitary pulmonary nodules with dynamic contrastenhanced MR imaging: a promising technique. Magn Reson Imaging 1995;13:923933.

20 Guckel C, Schnabel K, Deimling M, Steinbrich W: Solitary pulmonary nodules: MR evaluation of enhancement patterns with contrast-enhanced dynamic snapshot gradient-echo imaging. Radiology 1996;200:681686.
21 Ohno Y, Hatabu H, Takenaka D, Adachi S, Kono M, Sugimura K: Solitary pulmonary nodules: potential role of dynamic MR imaging in management initial experience. Radiology 2002;224:503-511.

-22 Padhani AR, Husband JE: Dynamic contrast-enhanced MRI studies in oncology with an emphasis on quantification, validation and human studies. Clin Radiol 2001;56: 607-620.

23 Swensen SJ, Viggiano RW, Midthun DE, Müller NL, Sherrick A, Yamashita K, Naidich DP, Patz EF, Hartman TE, Muhm JR, Weaver AL: Lung nodule enhancement at CT: multicenter study. Radiology 2000;214: 73-80.

24 Zhang M, Kono M: Solitary pulmonary nodules: evaluation of blood flow patterns with dynamic CT. Radiology 1997;205:471-478.

-25 Fraioli F, Anzidei M, Zaccagna F, Mennini ML, Serra G, Gori B, Longo F, Catalano C, Passariello R: Whole-tumor perfusion CT in patients with advanced lung adenocarcinoma treated with conventional and antiangiogenetic chemotherapy: initial experience. Radiology 2011;259:574-582.

26 Ohno Y, Koyama H, Matsumoto K, Onishi Y, Takenaka D, Fujisawa Y, Yoshikawa T, Konishi M, Maniwa Y, Nishimura Y, Ito T, Sugimura K: Differentiation of malignant and benign pulmonary nodules with quantitative first-pass 320-detector row perfusion CT versus FDG PET/CT. Radiology 2011; 258:599-609.

27 Henzler T, Schmid-Bindert G, Schoenberg SO, Fink C: Diffusion and perfusion MRI of the lung and mediastinum. Eur J Radiol 2010;76:329-336.

28 Pauls S, Mottaghy FM, Schmidt SA, Krüger S, Möller P, Brambs HJ, Wunderlich A: Evaluation of lung tumor perfusion by dynamic contrast-enhanced MRI. Magn Reson Imaging 2008;26:1334-1341 